\title{
Emergency Cervical Internal Carotid Artery Stenting in Combination with Intracranial Thrombectomy in Acute Stroke
}

\author{
S. Stampfl, P.A. Ringleb, M. Möhlenbruch, C. Hametner, C. Herweh, M. Pham, J. Bösel, S. Haehnel, M. Bendszus, and S. Rohde
}

\begin{abstract}
BACKGROUND AND PURPOSE: In past years, thrombectomy has become a widely used procedure in interventional neuroradiology for the treatment of acute intracranial occlusions. However, in $10-20 \%$ of patients, there are additional occlusions or stenotic lesions of the ipsilateral cervical internal carotid artery. The purpose of this study was to evaluate the feasibility of emergency carotid artery stent placement in combination with intracranial thrombectomy and the clinical outcome of the treated patients.
\end{abstract}

MATERIALS AND METHODS: We analyzed clinical and angiographic data of patients who underwent emergency cervical ICA stent placement and intracranial thrombectomy with stent-retriever devices in our institution between November 2009 and July 2012 . Recanalization was assessed according to the Thrombolysis in Cerebral-Infarction score. Clinical outcome was evaluated at discharge (NIHSS) and after 3 months (mRS).

RESULTS: Overall, 24 patients were treated. The mean age was 67.2 years; mean occlusion time, 230.2 minutes. On admission, the median NIHSS score was 18. In all patients, the Thrombolysis in Cerebral Infarction score was zero before the procedure. Stent implantation was feasible in all cases. In 15 patients (62.5\%), a Thrombolysis in Cerebral Infarction score $\geq 2$ b could be achieved. Six patients (25\%) improved $\geq 10$ NIHSS points between admission and discharge. After 90 days, the median mRS score was 3.0. Seven patients (29.2\%) had a good clinical outcome (mRS 0-2), and 4 patients (16.6\%) died, 1 due to fatal intracranial hemorrhage. Overall, symptomatic intracranial hemorrhage occurred in 4 patients $(16.6 \%)$.

CONCLUSIONS: Emergency ICA stent implantation was technically feasible in all patients, and the intracranial recanalization Thrombol$y$ sis in Cerebral Infarction score of $\geq 2 b$ was reached in a high number of patients. Clinical outcome and mortality seem to be acceptable for a cohort with severe stroke. However, a high rate of symptomatic intracranial hemorrhage occurred in our study.

ABBREVIATION: ECASS = European Cooperative Acute Stroke Study

S tent-retriever devices play an increasing role in the treatment of acute ischemic stroke, ${ }^{1-3}$ providing a high recanalization rate and a better functional outcome ${ }^{4}$ compared with other recanalization devices. In approximately $10 \%-20 \%$ of patients with stroke, ${ }^{5}$ additional ipsilateral high-grade ICA stenosis is present, which further complicates endovascular access and may lead to a delay in recanalization of the target vessel occlusion. On the other hand, patients with high-grade ICA stenosis might profit from pre-existing intracranial collaterals that-theoretically-can ex-

\section{Received May 24, 2013; accepted after revision August 6.}

From the Departments of Neuroradiology (S.S., M.M., C. Herweh, M.P., S.H., M.B., S.R.) and Neurology (P.A.R., C. Hametner, J.B.), University of Heidelberg, Heidelberg, Germany.

Please address correspondence to Sibylle Stampfl, MD, Im Neuenheimer Feld 400 , 69120 Heidelberg, Germany; e-mail: sibyllestampfl@gmx.de

三 Indicates article with supplemental on-line table.

http://dx.doi.org/10.3174/ajnr.A3763 tend the time window for endovascular treatment in terms of beneficial clinical outcome. However, this hypothesis so far remains unproven either by experimental or clinical studies.

We prospectively collected clinical and angiographic data of patients with acute cerebral vessel occlusions of the anterior circulation and ipsilateral high-grade atherosclerotic stenosis or occlusion of the proximal/cervical ICA who underwent emergency ICA stent placement and intracranial thrombectomy with stentretriever devices. We present the experience at our institution between November 2009 and July 2012.

\section{MATERIALS AND METHODS}

\section{Inclusion Criteria and Patient Selection}

Approval for prospective collection of clinical and interventional data was granted by the institutional review board.

We analyzed prospectively collected data of all patients with stroke who had embolic intracranial vessel occlusion of the anterior circulation and at the same time presented with atheroscle- 
rosis-related high-grade cervical ICA stenosis or ICA occlusion. Patients with arterial dissections were excluded from this analysis. The eventual therapeutic procedure after cervical stent placement was intracranial endovascular recanalization with stent-retriever devices.

On admission, CT/CTA or MR imaging/MRA, including diffusion and perfusion imaging, was performed to evaluate the extension of the ischemic lesion and the potential tissue at risk (penumbra). Patients were selected for endovascular stroke treatment if they had an NIHSS score of $\geq 10$ and CTA-proved vessel occlusion of the distal ICA or M1/2 segment and absence of early signs of an extensive and advanced infarction (more than one-third of the MCA territory) on CT- or MRA-proved vessel occlusion of the distal ICA or M1/2 segment, a DWI lesion less than one-third of the MCA territory, and/or a PWI/DWI mismatch of more than one-third on visual assessment.

\section{Evaluation of Pretherapeutic Imaging Data}

The above-mentioned CT and MR imaging inclusion criteria were noted. Furthermore, pretherapeutic CT or MR images were analyzed according to the ASPECTS study group. ${ }^{6,7}$

\section{Interventional Procedure}

All procedures were performed with the patient under general anesthesia. As vascular access, an $8 \mathrm{~F}$ sheath was placed in the right common femoral artery. Then, an $8 \mathrm{~F}$ guiding catheter was placed in the ipsilateral common carotid artery, and the cervical ICA stenosis was passed with a 0.014 -inch microwire. A self-expanding stent (eg, Wallstent; Boston Scientific, Natick, Massachusetts) was advanced to cover the stenosis. After stent implantation, balloon dilation (Maverick balloon dilation catheter; Boston Scientific) was performed. If necessary, the stenosis was predilated with a percutaneous transarterial angioplasty balloon to allow fast access to the intracranial vasculature. If passage of the stenosis with an intermediate catheter was possible without stent placement, stent implantation was performed after thrombectomy. In case of complete cervical ICA occlusion due to underlying atherosclerosis, the occlusion was first crossed with a 0.027 -inch microcatheter over a 0.014 -inch microwire; afterward, predilation and carotid artery stent placement were performed as described above. We did not observe any case of spontaneous intracranial recanalization in patients with ICA and MCA occlusion immediately after ICA stent implantation.

For intracranial thrombectomy, an intermediate catheter $(6 \mathrm{~F}$ Neuron catheter; Penumbra, Alameda, California) was advanced through the cervical ICA and over the stent and positioned in the distal ICA as close to the thrombus as possible. A 0.027-inch microcatheter (eg, Rebar-18; Covidien, Irvine, California) was advanced through the thrombus, and the tip was placed distal to the thrombus. Then, a stent-retriever device (Solitaire; Covidien; or Revive; Codman Neurovascular, Raynham, Massachusetts) was deployed by pulling back the microcatheter. For thrombectomy, the device and microcatheter were simultaneously pulled back under continuous aspiration through the Neuron catheter. In case of incomplete recanalization, thrombectomy was repeated.

\section{Evaluation of Angiographic Data}

Angiographic images were analyzed regarding the following data: pretherapeutic level of ICA stenosis according to the North American Symptomatic Carotid Endarterectomy Trial method, ${ }^{8}$ time between ICA stent implantation and flow restoration, time to flow restoration (time interval between the first diagnostic angiographic image and the first image with evidence of re-established perfusion within the occluded vessel segment), time to complete revascularization (time interval between the first diagnostic image and the completion angiogram), thrombus length, Thrombolysis in Cerebral Infarction score (score ranging from $0=$ no perfusion to $3=$ regular perfusion $^{9}$ ) before and after the procedure, number of stent passes necessary for intracranial recanalization, and procedure-related complications. Successful recanalization was defined as TICI $\geq 2 \mathrm{~b}$. All angiographic images were evaluated in consensus by 2 neuroradiologists (S.S., S.R.).

\section{Outcome Assessment}

Follow-up CT imaging was performed 24-36 hours after the procedure or whenever clinical worsening occurred. Hemorrhage was classified according to the European Cooperative Acute Stroke Study (ECASS)-2 classification. ${ }^{10}$

Patients were assessed clinically on admission (NIHSS), at discharge (NIHSS, mRS), and after 90 days (mRS). Early clinical improvement was defined as NIHSS improvement of at least 10 points. Good clinical outcome was defined as mRS 0-2 at day 90 . Outcome was assessed during an in-hospital visit or by a semistructured telephone interview by a neurologist not blinded to the initial treatment.

\section{Statistics}

Continuous data were collected in a data base and are described by median and interquartile range or as mean and SD in case of normal distribution. Statistical analysis was performed by GraphPad Prism 5.0 for OS X (GraphPad software, San Diego, California). For comparison of time to flow restoration in all patients with stroke and in patients with stroke with carotid stents, the nonparametric Mann-Whitney $U$ Test was applied. A $P$ value $<.05$ was statistically significant.

\section{RESULTS}

Overall, 136 patients with acute intracranial anterior circulation occlusion were treated between November 2009 and July 2012 by using stent-retriever devices. Twenty-four (17.6\%) of these patients had an additional high-grade proximal stenosis (12 patients) or occlusion (12 patients) of the ipsilateral cervical ICA. Twenty-one patients were treated with ICA stent implantation and subsequent intracranial thrombectomy. Three patients underwent these treatments in reverse order. Stent implantation was technically feasible in all cases (details of each patient are shown in the Table and On-line Table).

In 17 patients, the glycoprotein IIb/IIIa receptor antagonist tirofiban was started before ICA stent implantation and was administered for 24-48 hours while an overlapping medication with aspirin, $100 \mathrm{mg} /$ day, and clopidogrel, $75 \mathrm{mg} /$ day, was initiated. In 5 patients, a loading dose of aspirin and clopidogrel was administered instead of tirofiban, and partial thromboplastin 

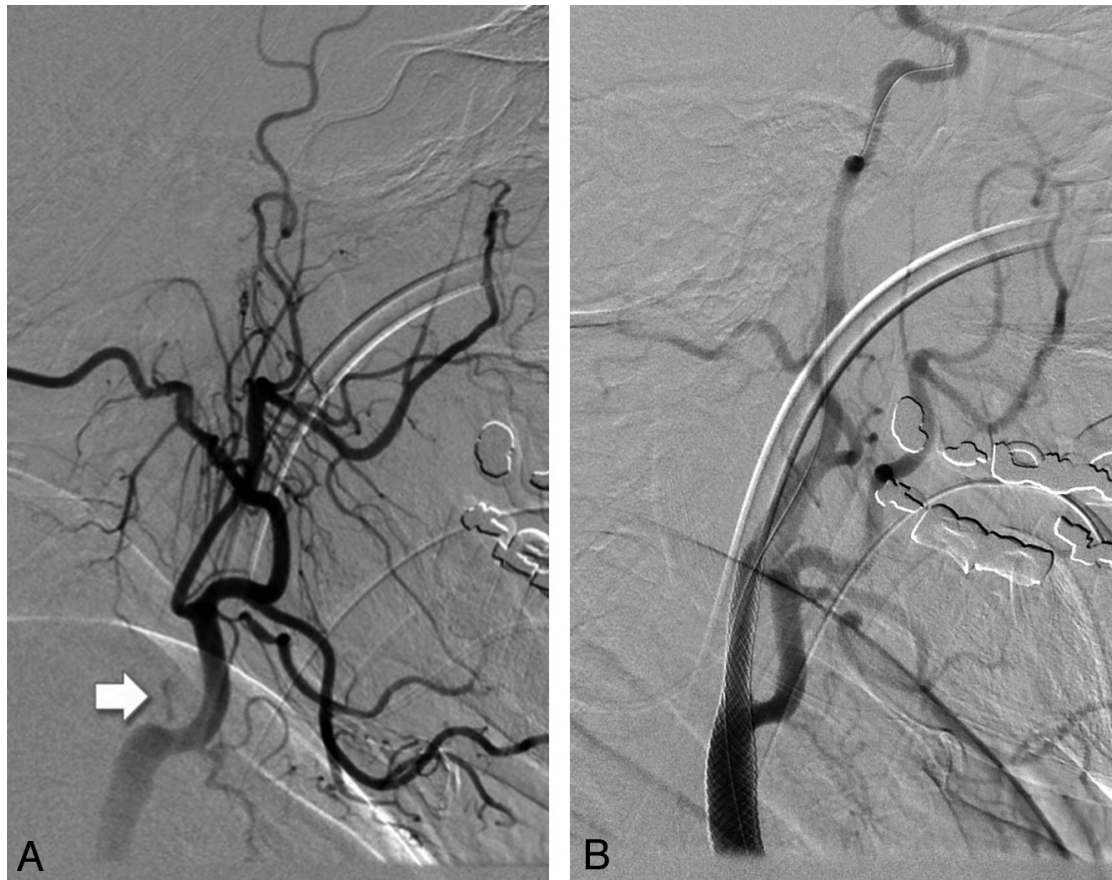

FIG 1. A, Occlusion of the cervical ICA close to the bifurcation (arrow). B, Successful reconstruction of the ICA lumen and sufficient antegrade flow after stent implantation and angioplasty.
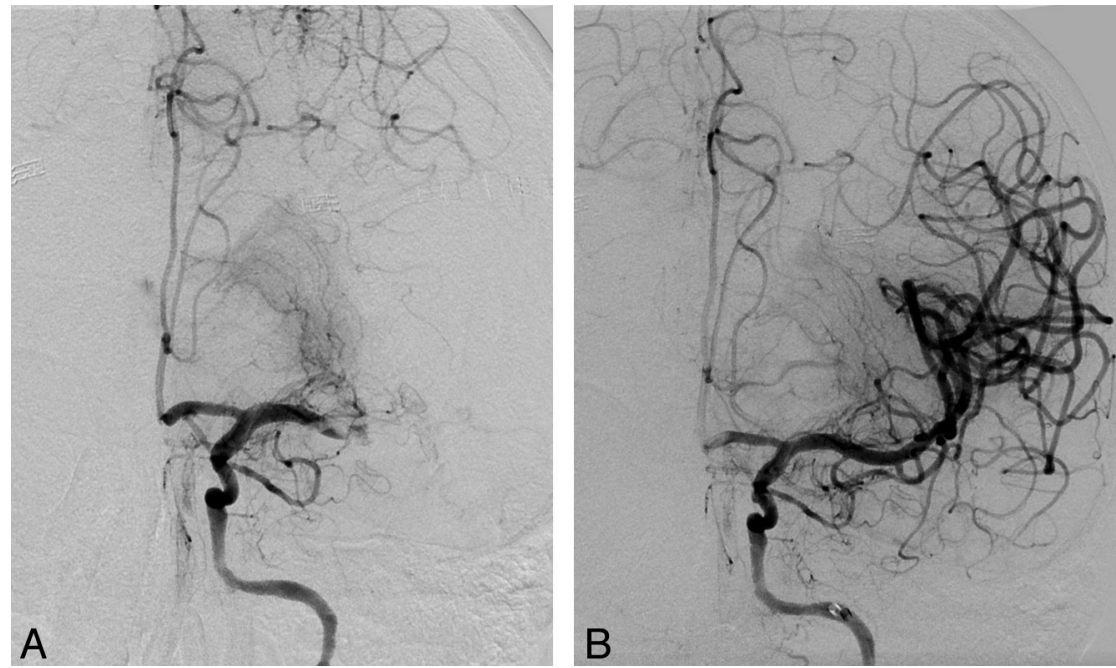

FIG 2. A, Intracranial angiogram shows a distal M1/M2 occlusion and good leptomeningeal collateralization via the anterior cerebral artery territory. $B$, After thrombectomy with the Revive device (1 device pass), complete recanalization of the M1/M2 segments could be achieved. Washout phenomenon in the anterior cerebral artery (A1 segment).

time-adjusted heparin was started. Two patients were already pretreated with aspirin and clopidogrel before the procedure, and additional heparin was initiated.

The mean age was $67.2 \pm 10.1$ years (range, 49-83; female/ male, $3: 21)$. The mean occlusion time was $230.2 \pm 131.3$ minutes (range, 134-643 minutes) in 15 patients. The patient with the longest occlusion time (643 minutes) presented with only mild symptoms at first (NIHSS 5), but his condition deteriorated within hours, despite IV thrombolysis, to NIHSS 12, and interventional recanalization was initiated. In 8 patients, the time of symptom onset was uncertain. In 1 patient, symptoms were mild for 3 days (NIHSS 4), and interventional recanalization was chosen after fluctuation and deterioration of his condition.

No patient had an ASPECTS score of $<7$ on pretherapeutic imaging evaluation. In all patients with CT imaging, early signs of infarction were mild and involved less than one-third of the MCA territory. In 1 patient undergoing MR imaging, the DWI lesion extent was estimated as one-third of the MCA territory, but with distinct PWI/DWI mismatch. In all other patients, the DWI lesion was less than one-third of the MCA territory. A PWI/DWI mismatch of more than one-third was found in all patients except 1 (in this patient, PWI was not performed due to agitation of the patient).

In all patients, cervical ICA changes visible on DSA resembled a typical atherosclerotic origin with various amounts of calcification, and acute stroke was caused by embolic occlusion of one of the major intracranial target vessels (MCA, $n=11$; carotid T/MCA, $n=13$ ).

In 22 patients, intravenous thrombolytic medication was administered prior to angiography according to the bridging concept ( $0.6 \mathrm{mg} / \mathrm{kg}$ body weight). In one of these patients, additional intra-arterial thrombolytic medication was injected through the microcatheter into an occluded MCA M2 branch. In 2 patients, only mechanical thrombectomy with stent-retriever devices and without bridging or intra-arterial lysis was performed.

Before the intracranial procedure, TICI was 0 in all patients. After the intervention, the median TICI was 2 (interquartile range, 2.0-3.0, [ie, TICI 0/1, $n=0$; TICI $2 \mathrm{a}, n=9$; TICI $2 \mathrm{~b}, n=4$; TICI 3, $n=11])$. Thus, in 15 patients (62.5\%), a TICI score of $\geq 2 b$ could be achieved. A median of 2 stent-retriever passes was necessary to achieve TICI 2b/3 (interquartile range, 1.0-2.0).

For recanalization, the Solitaire stent was used in 4 patients and the Revive device, in 17 patients; in 3 patients, both devices were used. In 1 patient, permanent Solitaire stent deployment was performed to achieve sufficient revascularization of the MCA.

Intracranial flow restoration was achieved after $53.4 \pm 20.5$ minutes (range, 20-100 minutes). The mean time to recanalization was $89.8 \pm 31.5$ minutes (range, $45-177$ minutes). The mean thrombus length was $15.9 \pm 8.3 \mathrm{~mm}$. Analysis of the time to flow restoration revealed that in patients who needed a carotid stent implantation, time to flow restoration was significantly longer 
Overview of the imaging criteria of each patient ${ }^{a}$

\begin{tabular}{|c|c|c|c|c|}
\hline Patient & $\begin{array}{l}\text { Vessel Occlusion } \\
\text { (CTA- or MRA-Proved) }\end{array}$ & $\begin{array}{l}\text { Early Signs of } \\
\text { Infarction Less Than } \\
\text { One-Third of the } \\
\text { MCA Territory on CT }\end{array}$ & $\begin{array}{l}\text { DWI Lesion Less } \\
\text { Than One-Third }\end{array}$ & $\begin{array}{l}\text { PWI/DWI Mismatch More } \\
\text { Than One-Third }\end{array}$ \\
\hline 1 & (MRA) & & + & + \\
\hline 2 & (CTA) & + & & \\
\hline 3 & (CTA) & + & & \\
\hline 4 & (MRA) & & + & + \\
\hline 5 & (CTA) & + & & \\
\hline 6 & (MRA) & & + & + \\
\hline 7 & (CTA) & + & & \\
\hline 8 & (MRA) & & + & + \\
\hline 9 & (CTA) & + & & \\
\hline 10 & (CTA) & + & & \\
\hline 11 & (CTA) & + & & \\
\hline 12 & (MRA) & & + & + \\
\hline 13 & (CTA) & + & & \\
\hline 14 & (MRA) & & + & + \\
\hline 15 & (CTA) & + & & \\
\hline 16 & (CTA) & + & & \\
\hline 17 & (MRA) & & + & + \\
\hline 18 & (CTA) & + & & \\
\hline 19 & (MRA) & & + & + \\
\hline 20 & (MRA) & & + & - \\
\hline 21 & (CTA) & + & & \\
\hline 22 & (MRA) & & & + \\
\hline 23 & (CTA) & + & & \\
\hline 24 & (CTA) & + & & \\
\hline
\end{tabular}

Note:- + indicates inclusion criteria met by patient; - indicates that no PWI was performed due to agitation of the patient.

${ }^{a}$ Imaging inclusion criteria for this analysis: All patients who were included in this analysis presented with an NIHSS score of $\geq 10$ ( 2 patients after clinical deterioration) and were treated with cervical ICA stent implantation and intracranial thrombectomy. Furthermore, they met the following imaging inclusion criteria: NIHSS score $\geq 10$ and CTAproved vessel occlusion of the distal ICA or Ml/2 segment and absence of early signs of an extensive and advanced infarction on CT, or MRA-proved vessel occlusion of the distal ICA or MI/2 segment, a DWI lesion less than $1 / 3$ of the MCA territory, and/or a PWI/DWI mismatch of more than $1 / 3$ on visual assessment.

than that in patients without stent implantation $(n=112)$, who underwent immediate thrombectomy with stent-retriever devices $(53.4 \pm 20.5$ minutes versus $33.8 \pm 23.7$ minutes; $P<.0001)$.

\section{Clinical Outcome}

On admission, the median initial NIHSS score was 18 (interquartile range, 15-22). Two patients (initial NIHSS scores of 4 and 5) were referred for interventional therapy only after fluctuation of symptoms and clinical deterioration. Six patients (25\%) improved $\geq 10$ NIHSS points between admission and discharge. After 90 days, the median mRS score was 3.0 (interquartile range, 2.0-5.0). Seven patients (29.2\%) had a good clinical outcome (mRS 0-2), 4 patients (16.6\%) died, 1 due to a fatal intracerebral bleeding. Among the patients with a good clinical outcome were 2 patients with TICI 2 a recanalization.

If one applied the definition of good neurologic outcome according to the SWIFT (Solitaire FR with the intention for thrombectomy) study $^{4}$ (mRS $\leq 2$ after 90 days, or NIHSS score improvement of $\geq 10$ points), 8 patients (33.3\%) met the criteria for a good neurologic outcome.

\section{Complications}

In one patient, extracranial internal carotid artery dissection distal to the ICA stent occurred after thrombectomy, which was treated with an additional Enterprise self-expanding stent implantation (Codman \& Shurtleff).

In 3 patients, distal thrombus embolization occurred during the thrombectomy maneuver with the stent retriever (migration of thrombus material into distal MCA branches and anterior cerebral artery branches, respectively). No distal embolic complications related to the ICA stent implantation were observed.

Symptomatic intracranial hemorrhage (classified according to the ECASS2 trial $^{10}$ ) occurred in 4 patients $(16.6 \%$; 3 had received tirofiban, 1 had received a loading dose of aspirin and clopidogrel) and was fatal in one of them.

\section{DISCUSSION}

In acute ischemic stroke, fast recanalization of the occluded vessel is probably the most important precondition for favorable clinical outcome. ${ }^{1,12}$ However, $\leq 20 \%$ of patients ${ }^{5}$ with intracranial vessel occlusion have additional high-grade cervical ICA stenosis or even total occlusion. Tandem cervical ICA/MCA occlusions are associated with low recanalization rates despite systemic thrombolysis and often have a poor outcome. ${ }^{13}$ Interventional procedures are a promising treatment option because a combination of ICA stent placement and intraarterial thrombolysis seems to improve the outcome. $^{14}$

To our knowledge, our study report represents the largest number of patients with stroke with cervical ICA stent placement and intracranial thrombectomy by using stent-retriever devices. Papanagiotou et $\mathrm{al}^{15}$ described their experiences with emergency carotid stent placement and intracranial thrombectomy procedures in 22 patients, but these authors in addition to stent-retriever devices (Solitaire stent) used other devices (Penumbra system) for the intracranial procedure.

Most recently, Matsubara et $\mathrm{al}^{16}$ published a report on 16 patients with acute cervical carotid occlusions caused by atherosclerosis, atrial fibrillation, or dissection. Ten of their patients had additional intracranial tandem occlusions. The patients were treated with various recanalization techniques. No stent retrievers were used. Similar to the present study, recanalization was successful in a high number of patients with cervical occlusion $(81.3 \%)$. In $43.8 \%$ of their patients, successful extracranial and intracranial flow was reached. Although one-third of their whole patient cohort presented with a favorable clinical outcome, only $20 \%$ of their patients with cervical and intracranial occlusion reached an mRS score of $0-2$, which is slightly lower than that in the present study. However, approximately $70 \%$ of their patients with successful extracranial and intracranial recanalization showed a favorable outcome, but none of the patients without successful extracranial or intracranial recanalization did. This finding clearly underlines the importance of technical success.

Malik et $\mathrm{al}^{17}$ reported their experience with recanalization procedures of 77 patients with tandem occlusion. In contrast to our study, they used various recanalization devices and techniques. A high recanalization rate $(75.3 \%)$ was reached. 
Thrombectomy with stent-retriever devices resulted in TICI $2 \mathrm{a}$ (partial filling of the entire vascular territory) in $37.5 \%$ of our patients despite several thrombectomy attempts; successful recanalization (corresponding to TICI $\geq 2 \mathrm{~b}$ ) was achieved in $62.5 \%$.

In agreement with the current literature, ${ }^{15-18}$ emergency ICA stent implantation was technically successful in all our patients. Not surprising, ICA stent implantation leads to a prolonged procedure time until recanalization. However, in our analysis, the treatment time was increased by only 20 minutes, which underlines the good technical feasibility of the stent-placement procedure.

In our series, we did not observe any case of spontaneous intracranial recanalization in patients with ICA and MCA occlusion immediately after ICA stent implantation, though this has been described in the literature as a common finding. ${ }^{17}$

Given that most patients had a severe stroke (median NIHSS score of 18), it is remarkable that the mortality rate was relatively low $(16.6 \%)$ compared with other series on tandem occlusion treatment (Malik et al, 24.7\%). ${ }^{17}$ However, $29.2 \%$ of our patients had an mRS of $0-2$ after 3 months, which is lower than that described by Malik et al (41.6\% good outcome). ${ }^{17}$ Twenty-five percent of our patients improved $\geq 10$ NIHSS points. If one applied the definition of good neurologic outcome according to the SWIFT study, ${ }^{4} 8$ patients $(33.3 \%)$ met the criteria for a good neurologic outcome.

Because distinct atherosclerotic ICA changes were present in all patients, the relatively high survival rate might be due to good collateral blood supply in patients with long-standing ICA stenosis. However, further preprocedural imaging data would be necessary to support this hypothesis.

In carotid artery stent placement, antithrombotic medication is administered to prevent acute stent thrombosis. However, it bears a potential risk of intracranial hemorrhage. The antithrombotic treatment concept differs in the literature. In the study of Matsubara et al, ${ }^{16}$ patients received a loading dose of aspirin and another antiplatelet drug, depending on the clinical conditions, if the postprocedural imaging showed no hemorrhage. The hemorrhage rate $(6.3 \%)$ was low. The hemorrhage rate $(10.4 \%)$ reported by Malik et $\mathrm{al}^{17}$ was slightly higher but still lower than that in our study. In their study, glycoprotein IIb/IIIa inhibitor was administered before stent placement, and an oral load of clopidogrel and aspirin was initiated if postprocedure imaging showed no hemorrhage. ${ }^{17}$ Later they changed their approach, and the patients received clopidogrel and aspirin before stent placement to avoid glycoprotein IIb/IIIa inhibition. However, they found no higher hemorrhage rate if patients were treated with glycoprotein IIb/ IIIa inhibitor.

In our series, intracranial hemorrhage occurred in a high proportion of patients $(16.6 \%)$, which was nearly triple the rates reported in the MR RESCUE ${ }^{19}$ and the Interventional Management of Stroke 3 trials. ${ }^{20}$ Although reperfusion trauma to a vascular territory that had been subjected to a long-term lower perfusion by the proximal stenosis might play a role, the high hemorrhage rate in our study might also be caused by the relatively aggressive antiplatelet therapy with full-dose tirofiban in most cases. Until
2012, our standard operation guidelines recommended the use of tirofiban when extracranial or intracranial stent placement was performed. Correspondingly, aspirin and clopidogrel were administered only in a minority of our patients. A recently published analysis of our patients with acute ischemic stroke in whom mechanical thrombectomy was performed revealed that additional treatment with tirofiban is associated with a worse outcome $e^{21}$ due to increased rates of fatal intracerebral hemorrhage. This finding caused us to rethink our treatment strategy. We now have revised our standard operation guidelines and give IV aspirin and clopidogrel via a gastric tube. Another option might be to defer the administration of antiplatelet therapy until the infarction size can be assessed on posttreatment imaging and the hemorrhage risk can be estimated. Alternatively, the interventionalist could even try to avoid emergency ICA stent implantation in the acute phase and perform only balloon angioplasty. Thus, no antiplatelet therapy associated with the risk of hemorrhage would be required.

In our series, in most of our patients, ICA stent implantation was performed before intracranial thrombectomy. Three patients were treated in the reverse order. In these cases, the ICA stenosis could be passed with the intermediate catheter after balloon dilation. However, ICA stent implantation became necessary because of persisting flow reduction within the extracranial ICA after successful intracranial thrombectomy. This so-called reverse technique (intracranial thrombectomy first, then extracranial ICA stent implantation) has been described recently by Cohen et al. ${ }^{22}$

\section{CONCLUSIONS}

Emergency ICA stent implantation was technically feasible in all our patients, corresponding to other studies on endovascular tandem-occlusion treatment, and intracranial recanalization TICI $\geq$ $2 \mathrm{~b}$ was reached in a high number of patients. The rate of favorable clinical outcome seems to be acceptable for such a cohort with severe stroke and is within the range of other endovascular stroke studies. However, in our series, a relatively high hemorrhage rate occurred.

Disclosures: Peter A. Ringleb-UNRELATED: Consultancy: Boehringer Ingelheim, Comments: less than $€ 500$ (\$665.04 US), Payment for Lectures (including service on Speakers Bureaus): Boehringer Ingelheim, Sanofi, PAION, Lilly, GlaxoSmithKline, Bayer Healthcare. Julian Bösel—UNRELATED: Payment for Lectures (including service on Speakers Bureaus): total \$5000, Comments: Covidien twice, talk on NIRS, satellite symposium; Sedana, twice, talk on AnaConDa Sedation Symposia; inviting hospitals or societies, 5 times; Payment for Manuscript Preparation: total $\$ 3000$, Comments: reviews for intensive up2date, review for Current Opinion in Cardiovascular Therapy congress syllabus for Neurocritical Care Society meeting, Travel/ Accommodations/Meeting Expenses Unrelated to Activities Listed: provided for several years by the medical societies (Deutsche Gesellschaft für Neurologie, German Neurological Society; Deutsche Gesellschaft für Neurointesiv- und Notfallmedizin, German Society for Neurological Intensive and Emergency Care; Deutsche interdisziplinäre Vereinigung für Intensivmedizin, German Interdisciplinary Association for Intensive Care; and the Neurocritical Care Society), Comments: accommodation and travel compensation when visiting conferences as an invited speaker, paid by inviting medical society (total $\$ 10,000$ ). Martin Bendszus-UNRELATED: Consultancy: Codman Neurovascular, Novartis, and Bayer Healthcare (clarify), Comments: speaker honoraria for educational talks, Other: principal investigator for RIVER 4 study (terminated, no recompensation), ${ }^{*}$ Codman Neurovascular.* Stefan Rohde-UNRELATED: Payment for Lectures (including service on Speakers Bureaus): Codman Neurovascular, AB Medica. *Money paid to the institution. 


\section{REFERENCES}

1. Castaño C, Dorado L, Guerrero C, et al. Mechanical thrombectomy with the Solitaire $\mathrm{AB}$ device in large artery occlusions of the anterior circulation: a pilot study. Stroke 2010;41:1836-40

2. Rohde S, Haehnel S, Herweh C, et al. Mechanical thrombectomy in acute embolic stroke: preliminary results with the Revive device. Stroke 2011;42:2954-56

3. Roth C, Papanagiotou P, Behnke S, et al. Stent-assisted mechanical recanalization for treatment of acute intracerebral artery occlusions. Stroke 2010;41:2559-67

4. Saver JL, Jahan R, Levy EI, et al. Solitaire flow restoration device versus the Merci retriever in patients with acute ischaemic stroke (SWIFT): a randomised, parallel-group, non-inferiority trial. Lancet 2012;380:1241-49

5. Grau AJ, Weimar C, Buggle F, et al. Risk factors, outcome, and treatment in subtypes of ischemic stroke: the German stroke data bank. Stroke 2001;32:2559-66

6. Barber PA, Demchuk AM, Zhang J, et al. Validity and reliability of a quantitative computed tomography score in predicting outcome of hyperacute stroke before thrombolytic therapy: ASPECTS Study Group-Alberta Stroke Programme Early CT Score. Lancet 2000;355:1670-74

7. Barber PA, Hill MD, Eliasziw M, et al. Imaging of the brain in acute ischaemic stroke: comparison of computed tomography and magnetic resonance diffusion-weighted imaging. J Neurol Neurosurg Psychiatry 2005;76:1528-33

8. Eliasziw M, Smith RF, Singh N, et al. Further comments on the measurement of carotid stenosis from angiograms: North American Symptomatic Carotid Endarterectomy Trial (NASCET) Group. Stroke 1994;25:2445-49

9. Higashida RT, Furlan AJ, Roberts $\mathrm{H}$, et al. Trial design and reporting standards for intra-arterial cerebral thrombolysis for acute ischemic stroke. Stroke 2003;34:e109-137

10. Hacke W, Kaste M, Fieschi C, et al. Randomised double-blind placebo-controlled trial of thrombolytic therapy with intravenous alteplase in acute ischaemic stroke (ECASS II): Second European-Australasian Acute Stroke Study Investigators. Lancet 1998;352:1245-51
11. Khatri P, Abruzzo T, Yeatts SD, et al. Good clinical outcome after ischemic stroke with successful revascularization is time-dependent. Neurology 2009;73:1066-72

12. Rha JH, Saver JL. The impact of recanalization on ischemic stroke outcome: a meta-analysis. Stroke 2007;38:967-73

13. Rubiera M, Ribo M, Delgado-Mederos R, et al. Tandem internal carotid artery/middle cerebral artery occlusion: an independent predictor of poor outcome after systemic thrombolysis. Stroke 2006;37:2301-05

14. Kwak HS, Hwang SB, Jin GY, et al. Predictors of functional outcome after emergency carotid artery stenting and intra-arterial thrombolysis for treatment of acute stroke associated with obstruction of the proximal internal carotid artery and tandem downstream occlusion. AJNR Am J Neuroradiol 2013;34:841-46

15. Papanagiotou $\mathrm{P}$, Roth $\mathrm{C}$, Walter $\mathrm{S}$, et al. Carotid artery stenting in acute stroke. J Am Coll Cardiol 2011;58:2363-69

16. Matsubara N, Miyachi S, Tsukamoto N, et al. Endovascular intervention for acute cervical carotid artery occlusion. Acta Neurochir (Wien) 2013;155:1115-23

17. Malik AM, Vora NA, Lin R, et al. Endovascular treatment of tandem extracranial/intracranial anterior circulation occlusions: preliminary single-center experience. Stroke 2011;42:1653-57

18. Jovin TG, Gupta R, Uchino K, et al. Emergent stenting of extracranial internal carotid artery occlusion in acute stroke has a high revascularization rate. Stroke 2005;36:2426-30

19. Kidwell CS, Jahan R, Gornbein J, et al. A trial of imaging selection and endovascular treatment for ischemic stroke. $N$ Engl J Med 2013;368:914-23

20. Broderick JP, Palesch YY, Demchuk AM, et al. Endovascular therapy after intravenous t-PA versus t-PA alone for stroke. $N$ Engl J Med 2013;368:893-903

21. Kellert L, Hametner C, Rohde S, et al. Endovascular stroke therapy: tirofiban is associated with risk of fatal intracerebral hemorrhage and poor outcome. Stroke 2013;44:1453-55

22. Cohen JE, Gomori M, Rajz G, et al. Emergent stent-assisted angioplasty of extracranial internal carotid artery and intracranial stentbased thrombectomy in acute tandem occlusive disease: technical considerations. J Neurointerv Surg 2013;5:440-46 